P229 (continued)

Outcome Measures and Analysis: Results were assessed using MANOVA, followed by univariate analyses, to identify if estimated consumption differed among survey weeks and whether results differed between treatment groups.

Results: The colorful plate group consumed more color variety than did the group that only tracked diet $(\mathrm{F}=8.144, \mathrm{df}=1,349, \mathrm{P}=0.005)$. However, there was no difference in the total amounts of fruit and vegetables consumed by the two groups $(\mathrm{P}=0.684)$. Both groups increased consumption of fruits and vegetables over the course of the study ( $\mathrm{P}=0.025$ for fruit and $\mathrm{P}=0.002$ for vegetables).

Conclusions and Implications: Tracking fruit and vegetables may create mindfulness of one's own eating habits, resulting in an increase in intake. Although the specific goal of "creating a colorful plate" did not increase the amount of fruits and vegetables consumed, it promoted eating habits likely to have added nutritional benefits.

Funding: None

\section{P230 The Effect of Descriptive Social Norms on Skin Carotenoid Scores and Fruit and Vegetable Intake in College Students}

HeidiWengreen, PhD, RD, LDN, heidi.wengreen@usu.edu, Utah State University, 8700 Old Main Hill, Logan, UT 84322; E. Nix, RD

Objective: Dietary behaviors, such as fruit and vegetable (FV) intake, may be influenced by the reported behavior of peers, known as the Descriptive Social Norm (DSN). The objective of the current study was to determine how DSN regarding skin carotenoid scores, a biomarker of fruit and vegetable intake, influences FV intake.

Design, Setting, Participants, and Intervention: Participants were recruited from an introductory nutrition course at Utah State University in 2015. Participants $(n=214)$ were randomly assigned to three groups. The control group received no information; the recommendation group received their skin carotenoid score and was informed that those meeting recommendations for $\mathrm{FV}$ had skin carotenoid scores of approximately 40,000; the DSN group received their skin carotenoid score and how their score compared to their peers.

Outcome Measures and Analysis: Carotenoid scans and frequency report of FV intake over the past week at baseline and 1 month after receiving the DSN information.

Results: Baseline averages for FV intake and skin carotenoid scores were 2.5 half-cup servings and 26,885 , respectively, and did not differ by group. Baseline FV intake was correlated to baseline skin carotenoid score $(r=.611$, $\mathrm{p}=.000$ ). Change variables were calculated and analyzed using ANOVA procedures for FV intake and skin carotenoids. No significant change was observed for either measure by group ( $\mathrm{p}=.709$ and $\mathrm{p}=.827$, respectively).

Conclusions and Implications: Presentation of actual DSN information had no effect on either FV intake or skin carotenoid score in this study. Future studies may evaluate longer time periods for change, or inclusion of an approval/disapproval (injunctive norm) message with DSN.

Funding: Utah Agriculture Experiment Station

\section{P231 Knowledge of Health Conditions Related to Drinking Sugar-Sweetened Beverage and Sugar-Sweetened Beverage Intake Among \\ U.S. Adults}

Sohyun Park,PhD, spark3@cdc.gov, Centers for Disease Control and Prevention, 4770 Buford Highway, NE, Mail Stop F-77, Atlanta, GA 30341; E. Lundeen, PhD;

H. M. Blanck, PhD

Objective: We examined associations between knowledge of health conditions related to drinking sugarsweetened beverages (SSBs) and SSB intake among adults.

Design, Setting, Participants, and Intervention: This cross-sectional study used the 2014 SummerStyles survey data for 4,163 U.S. adults ( $\geq 18$ years).

Outcome Measures and Analysis: The outcome measure was frequency of SSB intake from 4 sources (regular soda, fruit drink, sports/energy drink, sweetened coffee/ tea drink). Exposure measures were knowledge of health conditions related to drinking SSBs: weight gain, diabetes, cavities, high cholesterol, heart disease, and hypertension. Logistic regression was used to estimate adjusted odds ratios (OR) for consuming SSBs $\geq 2$ times/day.

Results: Overall, $37.8 \%$ of adults reported consuming SSBs $\geq 2$ times/day. Although most identified that weight gain $(80.2 \%)$, diabetes $(73.6 \%)$, and cavities $(71.8 \%)$ are related to drinking SSBs, fewer adults identified high cholesterol (24.1\%), heart disease (31.5\%), and hypertension (33.0\%) as related to drinking SSBs. Lower SSB intake was associated with knowledge that weight gain, diabetes, cavities, and heart disease are related to SSBs $(\mathrm{p}<0.001$, $\chi 2$ test). However, after adjustment for age, sex, race/ ethnicity, education, marital status, household income, weight status, and region, only reporting no knowledge that heart disease is related to drinking SSBs was significantly associated with consuming SSBs $\geq 2$ times/day (or $=1.29$ ).

Conclusions and Implications: Knowledge was low for some SSB-related health conditions. Our finding that knowledge of health conditions related to drinking SSBs, except heart disease, was not associated with SSB intake suggests that knowledge alone may not be sufficient for behavior change. Improving health education regarding adverse effect of high SSB intake may be a still important to lower SSB intake.

Funding: None 